\title{
The Feeling-of -knowing and Feeling-of-not-knowing Judgment under Different Levels of Processing and Memory Materials
}

\author{
based on a dual-process hypothesis
}

\author{
Yunyi Sun \\ Key laboratory of Behavioral Science, Institute of \\ Psychology, Chinese Academy of Sciences \\ University of China Academy of Sciences \\ Beijing, PR China \\ e-mail: 13910399594@139.com
}

\section{Xi Chen}

Key laboratory of Behavioral Science, Institute of Psychology, Chinese Academy of Sciences

University of China Academy of Sciences

Beijing, PR China

e-mail: chenx@psych.ac.cn

\author{
Ye Bai \\ Key laboratory of Behavioral Science, Institute of \\ Psychology, Chinese Academy of Sciences \\ University of China Academy of Sciences \\ Beijing, PR China \\ e-mail:leaf-103@163.com
}

Yiwen Chen

Key laboratory of Behavioral Science, Institute of Psychology, Chinese Academy of Sciences

Beijing, PR China

e-mail: chenyw@ psych.ac.cn

\begin{abstract}
Previous research implicated that feeling-ofknowing (FOK) and feeling-of-not-knowing (FOnK) might be two different cognitive processes. Based on this hypothesis, an experiment was conducted by examining FOK and FOnK judgment under different levels of processing and different kinds of materials. The results showed that processing depth had positive effects on FOK judgment, but little effects on FOnK judgment, conversely, it even decreased the accuracy of FOnK judgment, which demonstrated that FOK judgment and FOnK judgment were two different cognitional processes. Implications and limitations are discussed.
\end{abstract}

Keywords- feeling-of-knowing; feeling-of-not-knowing; processing level; memory materials; metacognition

\section{INTRODUCTION}

Metacognitive judgments of knowing a response or being able to later recognize a response are referred to as feeling-of -knowing (FOK) judgments, an essential aspect of memory self-monitoring insofar as such judgments bear directly on decisions to initiate, sustain, and eventually terminate a search of long-term memory (Singer \& Tiede [1]). A FOK can arise even when an item cannot be recalled, and people can predict what they can recall that item in the future (e.g., Hart [2]). Empirically, this feeling of knowing (FOK) has been examined by having participants predict subsequent recognition memory performance on items that were initially encoded but that cannot presently be recalled (Thomas, Bulevich, \& Dubois [3]).

Much research has been devoted to the factors determining the magnitude and accuracy of FOK judgment, such as time interval after remembering, target presenting time, accessible cues, learning level, etc. (e.g. Chan \&
McDermott [4]; Hanczakowski, Zawadzka, \& CockcroftMcKay [5]).

The present study examined the influence of processing depth and memory material on the FOK and FOnK judgment. Previous study argued that processing depth had an effect on FOK judgment. Lupker, Harbluk, \& Patrick [6] found that in high, moderate, low, there different levels of processing, the accuracy of FOK judgment increased as the processing level deeper. Memory materials also had an effect on FOK judgment. Many different kinds of materials had been examined, in order to have a better understand of FOK judgment, such as words, graphic, face, song and so on (e.g. Li, 2000 [7], Rabinovitz \& Peynircioğlu [8]), showing the impact of material attributes on FOK judgment. But these researches above are lack of a comprehensive analysis of the relationship between these factors.

Luo, Kazuhisa, Ying, \& Luo [9] conducted an fMRI study that brought up the dual-process hypothesis of FOK and FOnK. Through neuro-image method, this research revealed how the brain could realize this function. Frontal activities, in particular left superior, inferior, and middle frontal gyrus, were observed to be associated in this metamemory process. The results also implied that feelingof-knowing and feeling-of-not -knowing could be mediated by the distinct neural and cognitive processes. Another theory applied in the present study is the accessibility model. According to the accessibility model, FOK judgments are determined by the amount of partial contextual information accessed, regardless of its correctness (Koriat [10]).

A 2 (Level of processing: shallow vs. deep) $\times 2$ (memory material: Chinese character vs. Pinyin) mixed factorial design was conducted, where encoding orientation was a within-subjects factor and material kind was a between-subjects factor. The classic paradigm recall - 
judgment-recognition (RJR) paradigm (Hart [2]) was applied in the present study.

We hypothesized that: $\mathrm{H} 1$, processing depth has a main effect on FOK rating and accuracy; $\mathrm{H} 2$, memory material has a main effect on FOK rating and accuracy; H3, there is an interaction effect between processing depth and memory materials on FOK and FOnK judgment.

\section{METHOD}

Wherever Times is specified, Times Roman or Times New Roman may be used. If neither is available on your word processor, please use the font closest in appearance to Times. Avoid using bit-mapped fonts if possible. True-Type 1 or Open Type fonts are preferred. Please embed symbol fonts, as well, for math, etc.

\section{A. Participants}

We analyzed data from 48 senior high school students in grade one (mean age $=16,24$ females and 24 males). Chinese is the first language of all of them. Participants were recruited randomly. Only individuals with normal acuity or normal corrected visual acuity were eligible to participate.

\section{B. Materials}

The template is used to format your paper and style the text. All margins, column widths, line spaces, and text fonts are prescribed; please do not alter them. You may note peculiarities. For example, the head margin in this template measures proportionately more than is customary. This measurement and others are deliberate, using specifications that anticipate your paper as one part of the entire proceedings, and not as an independent document. Please do not revise any of the current designations.

One kind of material in the present study is Chinese character. We selected 384 Chinese characters from Modern Chinese frequency dictionary [11]. Only the Chinese characters with neutral emotion value, stroke number from 8 to 13 and frequency from 0.01 to 0.09 were selected as target items. We divided them into two groups. Each of the groups contains 192 Chinese characters. For the learning phase, we constructed 36 cue-target pairs randomly by using 72 Chinese characters in one group. In each pair, the left Chinese character was as cue item, and the right one as target item (e.g. 标一插). The very first 3 pairs and last 3 pairs in these 36 pairs presented to the participants were as buffer items and did not calculated into the results so as to avoid primacy and recency effects. For the recognition test, we used a five-alternative forced-choice test. Distracters were selected from those remain 120 Chinese characters. Every 4 Chinese characters in those remain 120 Chinese characters plus one of the 30 pairs' target items (without the buffer items) consist of five Chinese characters alternatives.

The other kind of material is Pinyin of Chinese characters. The selection procedure of Pinyin is a simulation of the Chinese character items selection procedure, except that changing the chosen Chinese characters in this part into their corresponding Pinyin. And the number of these Pinyin letters was controlled from 3 to 5 .

\section{Procedure}

The experiment was conducted with E-Prime software on Windows-based personal computers. The study description indicated that we were investigating how information processing differences affect both the way information is stored and how accessible that information becomes for later use. The participants were divided into two groups and involved in two parts of the experiment respectively. One part is for the Chinese character material, and the other is for the Pinyin material. Both parts followed the RJR paradigm. In each part, two sessions were conducted. These two sessions were distinguished by the depth of processing which is manipulated by the experiment instructor toward participants. Session 1 was conducted as shallow processing and session 2 deep processing. 10 minutes' rest was taken between the two sessions, and the order of the two sessions was counterbalanced among participants in both parts. There were four phases in each entire session: learning phase, cuedrecall phase, FOK and FOnK judgments phase and recognition phase. Participants read the instructions for each phase on the computer.

\section{1) Part I Chinese character materials}

a) Learning phase. In this phase, we manipulated levels of processing at encoding within participants. During the encoding phase, we presented participants with cuetarget pairs, one pair at a time for 6 seconds, and asked them to answer one of the following orienting questions for each pair: in session 1, "whether if at least one Chinese character in the pair has the Chinese character component $f$ ?", or in session 2, "Could you make a sentence that contains both of the present Chinese characters?", and then to do the $\mathrm{Y} / \mathrm{N}$ choice. The component question was designed to promote "shallow" processing of the cue-target pairs, while the sentence-making question was designed to promote "deep", processing of the cue-target pairs. Each pair presented to participants for 6 seconds and the 36 cue-target pairs were presented. When the presenting was over, the participants were asked to execute the Down-minus-3 task to avoid the rehearsal and recency effects.

b) Cue-recall phase. In this phase, we presented participants with only the cues from each cue-target pair and asked them to produce the target by typing a word into the response box.

c) FOK and FOnK judgments phase. After recalling phase, participants were presented with cue words, which were not recalled correctly in cue-recall phase and asked to choose either FOK judgment or FOnK judgment correctly as soon as possible. FOK indicated that participants could not recall the corresponding target word, but some impression of the word that could recognition it from some alternatives. FOnK indicated that participants had no impression of the target word and could either recall or recognition it. If the participants chose the FOK judgment, they were then asked to rate their feeling of knowing for the target item. We asked participants to predict their chances of recognizing the target out of five choices when presented 
with the cue in a later phase of the experiment. Responses to the FOK question ("what are your chances of recognizing the correct target?") were provided on a 100-point scale (1 = I definitely will NOT be able to recognize the target word/image; $100=$ I definitely WILL be able to recognize the target word/image). The presenting duration of the cue word is two minutes; the next cue word would take place of the present one even if the participants did not respond to the prediction.

d) Recognition phase. Finally, participants completed a forced-choice recognition test. Participants viewed the cues from each stimuli pair (presented in a random order) and selected the corresponding target item from five alternatives. Participants responded using the number keys on the keyboard. Following the experiment, participants were debriefed and thanked for their participation.

\section{2) Part II Pinyin materials}

The second part used Pinyin materials by the other group of participants which were different from whom participant in the part I. However, the procedure of this part is also a simulation of the first part. The difference was the encoding manipulation by instructions presented to the participants in learning phase. In this part's learning phase, we manipulated levels of processing at encoding within participants by asking them: in session 1, "whether if the sum total number of the alphabet $a$ in the word pair could be above 2?" or ins session 2, "please spell out the corresponding Chinese character of each Pinyin word presents, and judge that if you could make a sentence that contains both of the present Chinese characters?", and then to do the Y/N choice. The number counting question was designed to promote "shallow" processing of the cue-target pairs, while the sentence-making question was designed to promote "deep" processing of the cue-target pairs.

\section{RESULTS}

\section{A. Cued recall and recognition}

For session 1 which used Chinese characters as materials, the cued recall performance averaged 0.01 correct for shallow processing and 0.33 correct for deep processing; for session 2 which used Pinyin materials, the cue recall performance averaged 0.00 for shallow processing and 0.21 for deep processing. A 2 (level of processing: shallow, deep) $\times 2$ (memory material: Chinese character, Pinyin) mixed analysis of variance (ANOVA) with level of processing as a within-participants factor and memory material as a between participants factor was performed to examine cued recall performance. The effects of level of processing and memory material were significant. A main effect of level of processing was found, $F(1,24)=147.78, p<0.001$. The response correctness under deep level of processing was significantly higher than shallow level of processing, which demonstrated the validity of this manipulated variable. In addition, a main effect of memory material was also found, $F$ $(1,24)=6.24, p<0.01$. The response correctness under Chinese character materials was significantly higher than Pinyin materials.

\section{B. Ratings of FOK judgments}

FOK judgments ratings refers to participants' confidence score of recognizing the target out of five choices when presented with the cue on a 100-point scale. A 2 (level of processing: shallow, deep) $\times 2$ (memory material: Chinese character, Pinyin) mixed analysis of variance (ANOVA) with level of processing as a within-participants factor and memory material as a between participants factor was performed to examine the ratings of FOK judgments. The mean score of FOK judgments ratings under different level of processing and memory materials are summarized in Table 1. A significant effect of interaction effect was found between level of processing and memory material, $F(1,24)$ $=10.08, p<0.01$. As can be seen in Figure 1, simple effect analyses revealed that there was no significant difference of FOK judgments ratings between two different memory materials under shallow processing; but under deep processing, the FOK judgments ratings of Chinese character was significantly higher than Pinyin $(p<0.01)$. And also, both of the memory materials' FOK judgments ranked higher in deep processing condition than in shallow processing $(p<0.001)$.

\section{Accuracy of FOK predictions and FOnK predictions}

Based on the performance in the FOK and FOnK judgments phase and the recognition phase, the result items were sorted into five types: SC( successful cue-recall), PP( positive FOK, positive recognition), NP ( negative FOK, positive recognition), PN, and NN: The predictive accuracy of FOK ( PA-FOK) and that of FOnK ( PA-FOnK) were estimated as follows (Luo, Kazuhisa, Ying, \& Luo [9]):

$$
\mathrm{PA}-\mathrm{FOK}=\mathrm{PP} / \mathrm{PP}+\mathrm{PN} .
$$

$$
\mathrm{PA}-\mathrm{FOnK}=\mathrm{NN} / \mathrm{NN}+\mathrm{NP} .
$$

TABLE I. MEAN FOK JUDGMENTS RATINGS SCORES UNDER DIFFERENT CONDITIONS

\begin{tabular}{lcccccc}
\hline Level of Processing & \multicolumn{3}{c}{ Shallow processing } & \multicolumn{3}{c}{ Deep processing } \\
Material & $\mathrm{N}$ & $M$ & $S D$ & $\mathrm{~N}$ & $M$ & $S D$ \\
\hline Chinese character & 22 & 23.93 & 8.29 & 22 & 72.18 & 18.39 \\
Pinyin & 14 & 24.50 & 14.49 & 14 & 47.98 & 22.14 \\
\hline
\end{tabular}

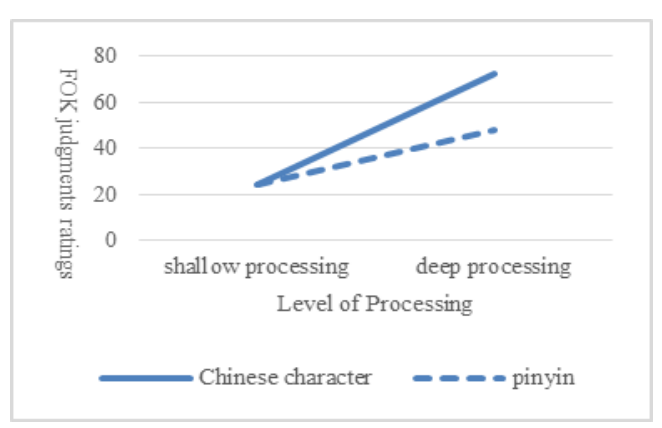

Figure 1. The interaction effect of level of processing and memory material on ratings of FOK judgments 
The result of the accuracy of FOK predictions and FOnK predictions calculated by the present method was showed following (see Table II).

TABLE II. THE ACCURACY OF FOK PREDICTIONS AND FONK PREDICTIONS

\begin{tabular}{llcccccc}
\hline & Level of processing & \multicolumn{3}{c}{ Shallow processing } & \multicolumn{3}{c}{ Deep processing } \\
& \multicolumn{1}{c}{ Material } & $\mathrm{N}$ & $\mathrm{M}$ & $S D$ & $\mathrm{~N}$ & $\mathrm{M}$ & $S D$ \\
\hline \multirow{2}{*}{ PA-FOK } & Chinese character & 22 & 0.37 & 0.18 & 22 & 0.74 & 0.16 \\
& Pinyin & 14 & 0.21 & 0.15 & 14 & 0.56 & 0.23 \\
\multirow{3}{*}{ PA-FOnK } & Chinese character & 22 & 0.68 & 0.19 & 22 & 0.68 & 0.79 \\
& Pinyin & 24 & 0.81 & 0.10 & 24 & 0.67 & 0.19 \\
\hline
\end{tabular}

A 2 (level of processing: shallow, deep) $\times 2$ (memory material: Chinese character, Pinyin) mixed analysis of variance (ANOVA) with level of processing as a withinparticipants factor and memory material as a between participants factor was performed to examine PA-FOK. There was no significant interaction effect between material and level of processing. A main effect of memory material was found, $F(1,24)=16.19, p<0.001$, comparisons revealed that FOK accuracy was higher when memorized Chinese character than Pinyin; a main effect of level of processing was also found, $F(1,24)=67.22, p<0.001$, comparisons revealed that FOK accuracy was higher under deep processing condition than shallow processing.

A 2 (level of processing: shallow, deep) $\times 2$ (memory material: Chinese character, Pinyin) mixed analysis of variance (ANOVA) with level of processing as a withinparticipants factor and memory material as a between participants factor was performed to examine PA-FOnK. But no significant interaction effect or main effects were found.

\section{DISCUSSIONS}

Our primary goal was to examine FOK judgment and FOnK judgment under different conditions of different processing depth and memory materials.

Main effect of processing depth, memory materials. Main effect were found under different processing depth, memory materials, which demonstrated H1 \& H2. The present study suggest that under deep processing conditions, participants not only had a better performance on recall, a higher FOK ratings, but also more accurate on FOK judgment, which indicated that FOK rating is determined by the amount of activated information as explained by the accessibility model (Koriat [10]). The result is in accord with the previous study: the accuracy of FOK judgment would increase under deep processing (Wang \& Luo [12]). Memory material had different effect on FOK and FOnK judgment. For FOK judgment, participants performed better in Chinese characters, which afford more cued information, than in Pinyin.

Interaction effect between processing depth and memory materials. This results demonstrated $\mathrm{H} 3$ and indicated that memory material had an effect on people's memory and metamemory monitoring by different processing level. There was no significant difference between Chinese character and Pinyin material under shallow processing; but under deep processing condition, participants could obtained more information from Chinese character material's shape, tone, and meaning. These results implied that processing depth functions by the material attributes of the cognitive target.

The dual-process hypothesis. However, the accuracy of FOnK judgment was not significantly higher under deep processing. Due to the mean number, FOnK judgment accuracy was even lower under deep processing than shallow processing; memory material had different effect on FOK and FOnK judgment. This result implied that FOK and FOnK judgment may belong to two dissociable cognitive process, verifying the dual-process hypothesis ( $\mathrm{Liu}, \mathrm{Su}, \mathrm{Xu}$, \& Chan [13]): FOK judgment is based on the accessibility model, while FOnK judgment is based on the cue familiarity model.

Limitations and future research. One of the limitations of the present study is that better control of the memory material is available. Furthermore, only two of linguistic materials were examined in the study, the implications discussed should be carefully used in other kinds of cognitive material.

In future research, the recall based recognition (remember response) and the familiarity based recognition (know response) should be distinguished, in order to discuss FOK judgment more precisely.

\section{ACKNOWLEDGMENT}

The corresponding author is Yiwen Chen, who has walked us through all the stages of the writing of this thesis. Without his consistent and illuminating instruction, this paper could not have reached its present form. The available e-mail of corresponding author is chenyw@psych.ac.cn.

\section{REFERENCES}

[1] M. Singer and H. L. Tiede, "Feeling of knowing and duration of unsuccessful memory search," Memory \& cognition, vol. 36, 2008, pp. 588-597.

[2] J. T. Hart, "Memory and the feeling-of-knowing experience," Journal of educational psychology, vol. 56, 1965, pp. 208.

[3] A. K. Thomas, J. B. Bulevich, and S. J. Dubois, "An analysis of the determinants of the feeling of knowing," Consciousness and cognition, vol. 21, 2011, pp. 1681-1694.

[4] J. C. Chan and K. B. McDermott, "The testing effect in recognition memory: a dual process account," Journal of Experimental Psychology: Learning, Memory, and Cognition, vol. 33, 2007, pp. 431.

[5] M. Hanczakowski, K. Zawadzka, and C. Cockcroft-McKay, "Feeling of knowing and restudy choices," Psychonomic bulletin \& review, 2014, pp. 1-6.

[6] S. J. Lupker, J. L. Harbluk, and A. S. Patrick, "Memory for things forgotten," Journal of Experimental Psychology: Learning, Memory, and Cognition, vol. 17, 1991, pp. 897.

[7] T. G. Li, "Sensibility and response bias of words and pictures in feeling-of-knowing," Acta Psychologica Sinica, vol. 32, 2000, pp. 264-268.

[8] B. E. Rabinovitz and Z. F. Peynircioğlu, "Feeling-of-knowing for songs and instrumental music," Acta psychologica, vol. 138, 2011, pp. 74-84. 
[9] J. Luo, N. Kazuhisa, X. P. Ying and Y. J. Luo, "Knowing that You Know and Knowing that You don't Know: A fMRI Study on Feelingof-Knowing (FOK)," Acta Psychologica Sinica, vol. 36, 2004, pp. 426-433.

[10] A. Koriat, "How do we know that we know? The accessibility model of the feeling of knowing," Psychological review, vol. 100, 1993, pp. 609.

[11] Beijing language College language instruction institute, "Modern Chinese Frequency Dictionary (in Chinese)," Beijing: Beijing language college Press, 1986.
[12] P. P. Wang and J. Luo, "Feeling-of-knowing (FOK) and feeling-ofnot-knowing (FOnK): an experimental disassociation," Acta Psychologica Sinica, vol. 37, 2005, pp. 442-449.

[13] Y. Liu, Y. Su, F. Xu and R. C. Chan, "Two dissociable aspects of feeling-of-knowing: Knowing that you know and knowing that you do not know," The Quarterly Journal of Experimental Psychology, vol. 60, 2007, pp. 672-680. 